Erschienen in: Deppermann, Arnulf/Linke, Angelika (Hrsg.): Sprache intermedial. Stimme und Schrift, Bild und Ton. - Berlin/New York: de Gruyter, 2010. S. 69-96. (Jahrbuch des Instituts für Deutsche Sprache 2009), https://doi.org/10.1515/9783110223613.69

\author{
Elisabeth Reber / Elizabeth Couper-Kuhlen
}

\title{
Interjektionen zwischen Lexikon und Vokalität: Lexem oder Lautobjekt?
}

\begin{abstract}
Anstelle der herkömmlichen Wortklasse Interjektion plädiert dieser Beitrag für eine erweiterte Auffassung von Lautobjekten (hier auch ,Vokalisierungen' genannt), wie sie von tatsächlichen Sprechern in konkreten Interaktionszusammenhängen produziert werden. Fokussiert wird der Gebrauch von Lautobjekten als affektgeladene Reaktion auf eine Mitteilung im Gespräch. Anhand eines Korpus von natürlich vorkommenden englischen Alltagsgesprächen werden drei Thesen erläutert: (1) Manche Lautobjekte bilden Lexeme, deren Form eine konventionalisierte prosodische Lautgestalt einschließt, (2) Lautobjekte werden als Teil einer körperlichen Gestalt produziert und (3) manche nicht-lexikalisierten Lautobjekte können ähnliche Funktionen wie lexikalisierte Lautobjekte übernehmen. Zum Schluss wird auf die sprachtheoretische Relevanz von Lautobjekten eingegangen.
\end{abstract}

\section{Einführung}

Spätestens seit der Verbreitung von „sound words“ in der Chat- und Instant-messaging-Welt ist die Interjektionsforschung wieder modern geworden. Denn obwohl Comicbücher - und damit ihre Soundwörter - durch den Animationsfilm zunächst verdrängt wurden, genießen Interjektionen und sonstige „dazwischen geworfenen“ Einheiten jetzt im so genannten Netspeak eine regelrechte Renaissance. Tabelle 1 zeigt ein paar Beispiele, entnommen einer jüngst erschienenen Arbeit zum Instant Messaging (Kessler 2008).

Diese Beispiele werfen unseres Erachtens einige Fragen auf: Sind Interjektionen und „sound“-Wörter überhaupt Wörter? Und sind sie wirklich so chaotisch „dazwischen geworfen“, wie die Etymologie des Wortes Interjektion nahelegt? Welche Vokalisierungen gehören zu der Kategorie von Interjektion bzw. Lautobjekt und welche nicht? Und welchen Status haben diese im sogenannten Sprachsystem? Auf diese und ähnliche Fragen soll im Folgenden eingegangen werden.

\section{Was sind Interjektionen?}

Es gibt inzwischen eine beachtliche deutschsprachige Literatur zu dieser Frage, u.a. Ehlich (1986), Fries (2002), Kehrein/Rabanus (2001), Kowal/ O'Connell (Hg.) (2004), Nübling (2001, 2004), Schmidt (2001), DUDEN (2005). Wir wollen uns hauptsächlich an Nüblings Verständnis von Inter- 


\begin{tabular}{|c|c|}
\hline Soundwort & Beispiel aus dem Korpus \\
\hline hmmm & $\begin{array}{l}\text { Datum und Uhrzeit: 09/14/2006 03:31 PM } \\
\text { Sie haben eine Nachricht gesendet an C } \\
\text { hmmm aber }\end{array}$ \\
\hline hehe & $\begin{array}{l}\text { Datum und Uhrzeit: 12/10/2006 08:09 PM } \\
\text { Sie haben eine Nachricht gesendet an E } \\
\text { hehe ich auch ;-) }\end{array}$ \\
\hline haha & $\begin{array}{l}\text { Datum und Uhrzeit: 04/02/2007 02:40 PM } \\
\text { Sie haben eine Nachricht empfangen von E } \\
\text { haha }\end{array}$ \\
\hline hihi & $\begin{array}{l}\text { Datum und Uhrzeit: } 02 / 09 / 200708: 26 \mathrm{PM} \\
\text { Sie haben eine Nachricht empfangen von B } \\
\text { hihi }\end{array}$ \\
\hline höhö & $\begin{array}{l}\text { Datum und Uhrzeit: } 02 / 03 / 200706: 57 \mathrm{PM} \\
\text { Sie haben eine Nachricht empfangen von B } \\
\text { min Mii rockt rächt, höhö }\end{array}$ \\
\hline tsss & $\begin{array}{l}\text { Datum und Uhrzeit: } 09 / 28 / 200611: 55 \mathrm{AM} \\
\text { Sie haben eine Nachricht gesendet an C } \\
\text { du bist in ro, und gehts online? } \\
\text { tsss }\end{array}$ \\
\hline öhm & $\begin{array}{l}\text { Datum und Uhrzeit: } 12 / 16 / 200608: 39 \text { PM } \\
\text { Sie haben eine Nachricht gesendet an C } \\
\text { öhm zeit? }\end{array}$ \\
\hline ehm & $\begin{array}{l}\text { Datum und Uhrzeit: 10/09/2006 11:32 AM } \\
\text { Sie haben eine Nachricht empfangen } \\
\text { von G } \\
\text { ehm 8oder } 9 \wedge \text { A }\end{array}$ \\
\hline autsch & $\begin{array}{l}\text { Datum und Uhrzeit: } 12 / 29 / 2006 \text { 04:20 PM } \\
\text { Sie haben eine Nachricht gesendet an C } \\
\text { autsch }\end{array}$ \\
\hline hmpf & $\begin{array}{l}\text { Datum und Uhrzeit: 10/29/2006 09:46 PM } \\
\text { Sie haben eine Nachricht empfangen von B } \\
\text { hmpf }\end{array}$ \\
\hline grmbl & $\begin{array}{l}\text { Datum und Uhrzeit: } 12 / 18 / 2006 \text { 06:10 PM } \\
\text { Sie haben eine Nachricht gesendet an C } \\
\text { da waren scheins zwei kleinere faruen die } \\
\text { ziemlich üss gewesen seien (laut D) und D } \\
\text { sagte zu F hey schau die wären doch was } \\
\text { und F jäääh grmbl nein hmpf blabla }\end{array}$ \\
\hline
\end{tabular}

Tab. 1: Soundwörter (Kessler 2008, S. 27)

jektionen anschließen. Nach diesem Verständnis ist die Kategorie Interjektion eine prototypisch organisierte Kategorie, bei der sich die Mitglieder unterschiedlich nah zum oder entfernt vom prototypischen Zentrum der Kategorie positionieren. Die Zugehörigkeit einer Einheit zur Kategorie Interjektion kann somit graduell sein: Einheiten können mehr oder weniger ,Interjektionalität' vorweisen. Zur MINIMALDEFINITION einer Interjektion werden laut Nübling folgende konstitutive Merkmale gerechnet (Nübling 2004, S. 13):

- expressiver Ausdruck einer spontanen Emotion,

- Abwesenheit referentieller Bedeutung,

- Unflektierbarkeit,

- syntaktische Autonomie,

- eventuelles Vorhandensein onomatopoetischer Strukturen. 
Nach dieser Minimalbestimmung gehören zu den prototypischen Interjektionen vor allem EMPFINDUNGSWÖRTER wie ach, au, ih, igitt, pfui usw., jedoch nicht ikonische schallnachahmende Wörter wie tatütata, klingeling, miau oder Denotativa (,vollkonventionalisierte Lexeme mit stilistisch neutralem Wert') wie Knall oder Kuckuck.

Nübling (2004, S. 15) führt folgende (unvollständige) Liste prototypischer Interjektionen im Deutschen auf:

\begin{tabular}{|c|c|}
\hline$a b !$ & (Erstaunen, Überraschung, Schmerz) \\
\hline ach! & $\begin{array}{l}\text { (Bewunderung, Erstaunen, aber auch Enttäuschung, Ab- } \\
\text { lehnung) }\end{array}$ \\
\hline aha! & (Überraschung, Genugtuung) \\
\hline au(a)/autsch! & (Schmerz) \\
\hline bäh! & (Ekel) \\
\hline brrr! & (Kälte, Abscheu) \\
\hline$h m !$ & (viele Funktionen je nach Länge und Tonem) \\
\hline bihi! & (Schadenfreude) \\
\hline hoppla! & (Überraschung) \\
\hline$b u(c h) !$ & (Erschrecken) \\
\hline bui! & (Bewunderung, Überraschung) \\
\hline burra! & (Sieg, Freude) \\
\hline$i b !$ & (Ekel, Abscheu, Entsetzen) \\
\hline igitt! & (Ekel) \\
\hline jubu! & (Freude) \\
\hline$n a !$ & (Ungeduld, Ungehaltenheit) \\
\hline naja! & (Ratlosigkeit) \\
\hline nanu! & (Verwunderung, Befremden) \\
\hline$o i !$ & (Überraschung, Bewunderung) \\
\hline$o b !$ & (Betroffenheit, Enttäuschung, Bewunderung) \\
\hline obo! & (Ver-/Bewunderung, Erstaunen) \\
\hline oje! & (Enttäuschung, Mitleid, Verachtung)) \\
\hline pab! & (Verachtung, Geringschätzung) \\
\hline pfui! & (Ekel, Abscheu) \\
\hline phb! & (Gleichgültigkeit, Verachtung) \\
\hline pub! & (Erleichterung, auch Ekel bei Gestank) \\
\hline tja! & (Ratlosigkeit) \\
\hline uff! & (Erleichterung) \\
\hline uh! & (Angst) \\
\hline ui! & (Überraschung, Bewunderung) \\
\hline
\end{tabular}

Damit wird klar, dass es die sogenannten PRIMÄREN Interjektionen sind, die als prototypisch betrachtet werden. 


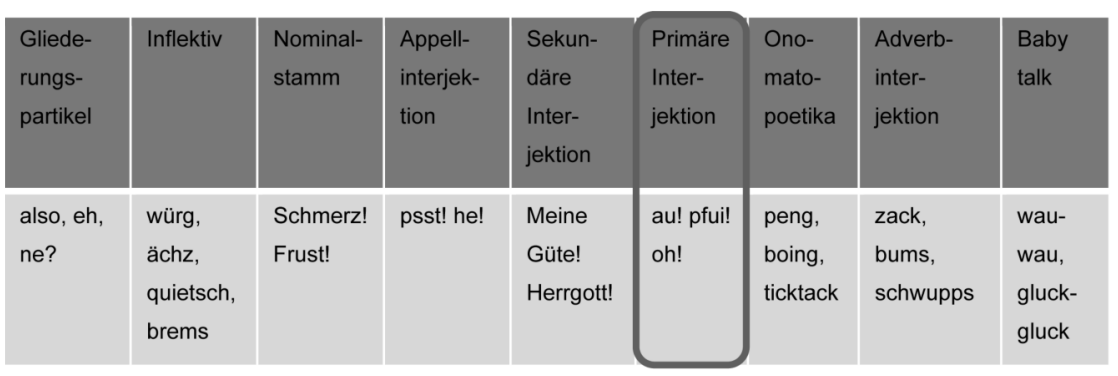

Tab. 2: Das interjektionale Spektrum (Nübling 2004, S. 34-35)

Ausgeschlossen aus der Kategorie der prototypischen Interjektionen sind sekundäre Interjektionen, ${ }^{1}$ die sich aus lexiko-syntaktischem Material entwickelt haben (meine Güte!, Herrgott!), sowie Appellinterjektionen bzw. Zurufe (psst! he!), Nominalstämme (Schmeræ! Frust!) und Inflektive (würg, quietsch). Weiterhin zählen Onomatopoetika (peng, boing), ,Baby talk' (wau-wau) und Adverbinterjektionen (zack, bums) nicht zu den prototypischen Interjektionen, genau so wenig wie Gliederungspartikel (also, ne, eh), weil sie nach Nübling keine - oder nur wenige - Emotionalität ausdrücken (siehe Tabelle 2). ${ }^{2}$

Doch welche EIGENSCHAFTEN haben Mitglieder der Kategorie der prototypischen primären Interjektionen? Im Folgenden sind einige in der Literatur rekurrente Merkmale aufgelistet:

1) Interjektionen sind NICHT REFERENTIELL. Mit Bühler (1982) haben Interjektionen statt einer Darstellungsfunktion eine Kundgabefunktion: sie stellen eine Befindlichkeitsmitteilung dar (Kehrein/Rabanus 2001, S. 47). Weinrich konstatiert: „der Sprecher [gibt mit ihrer Hilfe] mehr oder weniger intensiv eine Gefühlslage zu erkennen“ (2005, S. 859). „In Grenzfällen (Überraschung, Schmerz ...) dient [die Interjektion] der Triebabfuhr" (ebd., S. 857).

2) Interjektionen bilden SPRECHAKTE. Diese gehören wohl zu den Expressiva: „Hiermit teile ich Dir mit, dass ich traurig bin“. In Verbindung mit Sätzen sind Interjektionen als ,illokutive Indikatoren“ (Nübling 2004, S. 20) zu betrachten, d.h. sie tragen zur Illokution der Proposition bei (siehe auch die Diskussionen in Ameka 1992; Wharton 2003; Wilkins 1992).

$1 \quad$ Die Unterscheidung zwischen primären und sekundären Interjektionen geht auf Wundt (1907) zurück. Siehe weiterhin Reisigl (1999) zu sekundären Interjektionen.

2 Mit dem Kriterium der Emotionalität legt Nübling (2004) somit eine enge Definition des Interjektionsbegriffs vor, die auf die Antike zurückgeht (Ehlich 1986, S. 20). Ein weiter gefasstes Verständnis des Interjektionsbegriffs zeigt z.B. Fries (2002), indem er zwischen verschiedenen Teilklassen nach Emotionsausdruck (ach, ob), körperlichem Empfinden (aua, brr, uff) und Appellfunktion (hallo, prost, pst) unterscheidet (ebd., S. 656). 
3) Interjektionen können MONOLOGISCH verwendet werden. Ein Hinweis hierfür soll sein, dass sie auch in gesprächspartnerlosen Situationen geäußert werden können (Weinrich 2005, S. 257; Sornig 2002, S. 463). Kehrein/Rabanus (2001, S. 47) stellen eine vergleichbare These für Interjektionen auf, die als Diskurspartikel (z.B. ja, hm) verwendet werden: „Äußerungen von Diskurspartikeln, die primär [Symptomcharakter] tragen, beziehen sich unabhängig von der sequenziellen Gesprächsstruktur auf den Sprecher selbst." [Hervorhebung im Original].

4) Interjektionen sind phonologisch und prosodisch EXZENTRISCH. Zum einen können sie phonologische und phonotaktische Abweichungen vom deutschen Sprachsystem aufweisen, z.B. ohne Vokal vorkommen (psst) bzw. fremde Laute (brrr, tsk) oder Lautverbindungen ( $p f u i)$ enthalten. Zum anderen haben sie abweichend von sonstigen Wörtern im Deutschen und Englischen eine distinktive tonale Struktur. Ehlich (1986) stellt fünf unterschiedliche Tonverläufe bei den deutschen Interjektionen hm, ah, oh, ih, au, ei, oi, hoi und na fest, die jeweils mit kurzen, langen oder reduplizierten Silben vorkommen können. Tabelle 3 zeigt einen Überblick der für hm ausgemachten Töne und Silbenlängen (Ehlich 1986, S. 75):

\begin{tabular}{|c|c|c|c|}
\hline Fon & einfach & kurz & redupliziert \\
\hline$\gamma$ & $\begin{array}{l}\text { hm } \\
\text { IV }\end{array}$ & $\begin{array}{l}\text { hm' } \\
\quad \mathrm{IV}^{\mathrm{K}}\end{array}$ & hmhǹ̀ \\
\hline & hm & $\begin{array}{l}\mathrm{hm}^{\prime} \\
\quad \|^{\mathrm{K}}\end{array}$ & hmhm' \\
\hline - & hm̄̄ & $\mathrm{hm}^{-\mathrm{\prime}}$ & $*$ \\
\hline$V$ & hm̌ & $\mathrm{hm}^{\prime}$ & hmhm̌r \\
\hline$\wedge$ & $\mathrm{hm}_{2}$ & & \\
\hline
\end{tabular}

Tab. 3: Matrix der Eigenschaften von HM (Ehlich 1986, S. 75)

Jedoch: so überzeugend die bisherige linguistische Beschreibung der Wortklasse Interjektion sein mag, so kann sie doch nicht darüber hinwegtäuschen, dass bis heute recht wenig über den tatsächlichen Gebrauch dieser 
Objekte im Gespräch bekannt ist. Angesichts der heutigen Kenntnislage über die Verwendung von Sprache in Interaktionszusammenhängen plädieren wir hier für eine alternative Auffassung hinsichtlich der erwähnten Interjektionsmerkmale:

ad 1: REINE KUNDGABE? Goffmans Arbeiten zu „response cries“ (1978) veranschaulichen, dass mit Schmerzausrufen, Stöhnen bei Anstrengung, Überraschungslauten, Ekelausrufen, Jubellauten und dergleichen zwar momentane Gedanken und Gefühle lautlich kundgetan werden, jedoch werden diese in einer ritualisierten Art und Weise und als Teil eines „,social arrangement" produziert. Das Individuum hat seinen Auftritt in der Öffentlichkeit genug unter Kontrolle, um z.B. beim Anstoßen seines Zehs ein „Verdammt nochmal" in einem Kindergarten zu unterdrücken. Andererseits muss ein erfreutes „Oh“ beim Auspacken einer hässlichen Vase nichts über die wahren Gefühlsregungen des Geburtstagskinds über das Geschenk der Schwiegermutter aussagen. Diese und weitere empirische Beobachtungen lassen erhebliche Zweifel daran aufkommen, dass Interjektionen nur „eine Gefühlslage zu erkennen“ geben (Weinrich 2005, S. 859) oder zur reinen „Triebabfuhr" dienen (ebd., S. 857). Vielmehr ist es denkbar, dass sie auch für sozialkommunikative Zwecke produziert werden.

ad 2: SPRECHAKT? ILLOKUTIVER INDIKATOR? Solche Bezeichnungen legen nahe, dass Interjektionen, auch wenn sie multifunktional sind, eindeutige Emotionen ausdrücken. Wie unklar die Bedeutungen einzelner Interjektionen jedoch sind, zeigt gerade der Vergleich von Arbeiten, in denen Interjektionen mit spezifischen Emotionszuschreibungen aufgelistet werden: Während z.B. Weinrich (2005, S. 859) ab und ob als Ausdruck von neutraler oder positiver Überraschung klassifiziert, steht bei Nübling (2004, S. 15) $a h$ ! für Erstaunen, Überraschung, Schmerz und $o$ ! für Betroffenheit, Enttäuschung, Bewunderung. Die Kontextualisierungstheorie (Gumperz 1982; Auer/di Luzio (Hg.) 1992) konstatiert in diesem Zusammenhang, dass gerade nicht-referentielle sprachliche Zeichen als Hinweis auf mögliche Deutungsschemata fungieren. Sie lösen kontextabhängige Inferenzen aus, die im späteren Verlauf der Interaktion ausgehandelt, d.h. bestätigt oder verworfen werden. Nach dieser Theorie bestünde der Beitrag einer Interjektion darin, nicht kontextlos den inneren Zustand eines Sprechers, z.B. Ärger, kundzutun, sondern im sequenziellen Rahmen darauf hinzuweisen, dass Ärger ein mögliches Interpretationsschema für sein Verhalten bieten könnte.

ad 3: MONOLOGISCH? Im Verwendungszusammenhang, d.h. im fokussierten Gespräch (Goffman 1963) - auch im öffentlichen Selbstgespräch sind sprachliche Äußerungen genauso wie ihre Abwesenheit immer kommunikativ. Dies ist damit erklärbar, dass ein fokussiertes Gespräch aus einem strukturierten Geflecht von Beitragspositionen besteht. Je nach Aktivitätsbzw. Sequenztyp sind bestimmte Handlungen an bestimmten Stellen erwart- 
bar. Abweichungen von diesen Erwartbarkeiten sind rechenschaftspflichtig (accountable) und interpretierbar (Heritage 1984b). Wenn also eine bestimmte Interjektion an einer bestimmten Stelle im Gespräch produziert wird, wird sie im sequenziellen Rahmen bedeutsam, d.h. sie legt Inferenzen nahe, die es den Rezipienten ermöglichen, zu entscheiden, was als Nächstes getan werden soll. Zum Beispiel: Nach einer Mitteilung in englischen Alltagsgesprächen zeigt die Rezeption mit oh an, dass sich der Informationsstand des Rezipienten verändert hat (Heritage 1984a, S. 304). Die Mitteilung wird damit als möglicherweise vollständig behandelt. Im Gegensatz dazu wird mit der Produktion von yeah eine Fortsetzung der Mitteilung nahegelegt. $O b$ und yeah besitzen also verschiedene Folgeimplikationen: Während ob in Mitteilungssequenzen als ein „change-of-state token“ fungiert und potentiell sequenzterminierend ist, kann yeab sequenzexpandierend wirken (Heritage 1984a, S. 301-302).

ad 4: DISTINKTIVITÄT NUR DURCH TONVERLAUF UND DAUER? Die intuitive Annahme, dass die Produktion von Interjektionen durch eine bestimmte Intonationskontur und oft durch Längung gekennzeichnet ist, ist sicherlich richtig. Jedoch deuten Studien zur Prosodie im Gespräch darauf hin, dass nicht nur Tonhöhe und Dauer, sondern auch Lautstärke, Rhythmus, Tonhöhenumfang, Tonhöhenregister und Pause an der prosodischen Gestalt von sprachlichen Äußerungen maßgeblich beteiligt sein können (Couper-Kuhlen/Selting (Hg.) 1996; Auer/Couper-Kuhlen/Müller 1999; CouperKuhlen/Ford (Hg.) 2004). Hinzu kommen die in letzter Zeit als phonetisch propagierten Merkmale, z.B. die artikulatorische Einstellung, die artikulatorische Stärke und die Stimmqualität bzw. Phonationsart, die zu unterschiedlichen stimmlichen Effekten und Qualitäten führen können. Alle diese Merkmale tragen mit dazu bei, dass die Gestalt einer Interjektion (wieder)erkennbar und im Kontext interpretierbar wird.

ad 5: GRAPHISCHE BESCHRÄNKUNG? Auffällig bei den gängigen Listen von Interjektionen ist die Beschränkung auf Vokalisierungen, für die eine graphische Form (wenn auch unnormiert) existiert. Zudem wird durch die gängige Hinzufügung eines Ausrufezeichens suggeriert, dass Interjektionen als exklamative Äußerungen realisiert werden. Bei rein lautlichen Phänomenen nur von der graphischen Form auszugehen, deutet aber auf einen gewissen „written language bias“ (Linell 2005) hin. Unklar bei einem solchen graphembasierten Verfahren bleibt, wie viele (möglicherweise halbkonventionalisierte) Vokalisierungen OHNE graphische Form dadurch unentdeckt bleiben. Wie ertragreich ein Entdeckungsverfahren „von unten“, d.h. ausgehend von der lautlichen Seite der Rede, sein kann, zeigen die Befunde von Ward (2006) für das Englische, hier als Übersichtstabelle: ${ }^{3}$

3 Bei dieser Darstellung sind die phonetischen Formen der belegten Vokalisierungen durch folgende Erweiterungen der englischen Orthographieregeln dargestellt: „u/uu“ = Schwa, 
All conversational non-lexical sounds in the corpus, with numbers of occurrences

$\begin{array}{llll}\text { <clear-throat> 2 } & \text { hmmmmm 1 } & \text { okay 8 } & \text { unkay 1 } \\ \text { <tongue-click> 22 } & \text { hn 1 } & \text { okay 1 } & \text { unununu 1 } \\ \text { <click>neeu> 1 } & \text { hn-hn 1 } & \text { ooa 1 } & \text { uu 6 } \\ \text { <click>nuu 1 } & \text { huh 2 } & \text { ookay 1 } & \text { uuh 1 } \\ \text { <click>ohh 1 } & \text { I1 } & \text { oooh 1 } & \text { uum 6 } \\ \text { <click>yeahh 1 } & \text { iiyeah 1 } & \text { ooooh 1 } & \text { uumm 1 } \\ \text { <noisy-inhale> 1 } & \text { m-hm 2 } & \text { oop-ep-oop 1 } & \text { uun 1 } \\ \text { achh 1 } & \text { mm 2 } & \text { u-kay 1 } & \text { uuuh 1 } \\ \text { ah 6 } & \text { mm-hm 1 } & \text { u-uh 4 } & \text { uuuuuuu 1 } \\ \text { ahh 1 } & \text { mm-mm 1 } & \text { u-uun 1 } & \text { wow 1 } \\ \text { ai 1 } & \text { mmm 3 } & \text { uam 1 } & \text { yah-yeah 1 } \\ \text { am 1 } & \text { myeah 2 } & \text { uh 36 } & \text { ye 1 } \\ \text { aoa 1 } & \text { nn-hn 4 } & \text { uh-hn 2 } & \text { yeah 70 } \\ \text { aoo 1 } & \text { nn-nnn 1 } & \text { un-hn-uh-hn 1 } & \text { yeah-okay 1 } \\ \text { aum 5 } & \text { nu 1 } & \text { uh-huh 3 } & \text { yeah-yeah 1 } \\ \text { eah 1 } & \text { nuuuuu 1 } & \text { uh-mm 1 } & \text { yeahh 2 } \\ \text { ehh 1 } & \text { nyaa-haao 1 } & \text { uh-uh 2 } & \text { yeahuuh 1 } \\ \text { h-hmm 1 } & \text { nyeah 1 } & \text { uh-uhmmm 1 } & \text { yegh 1 } \\ \text { haah 1 } & \text { o-w 1 } & \text { uhh 4 } & \text { yeh-yeah 1 } \\ \text { hh 3 } & \text { oa 1 } & \text { uhhh 1 } & \text { yei 1 } \\ \text { hh-aaaah 1 } & \text { oh 20 } & \text { ukay 2 } & \text { yo 1 } \\ \text { hhh 1 } & \text { oh-eh 1 } & \text { um 20 } & \text { yyeah } \\ \text { hhh-uuuh 1 } & \text { oh-kay 1 } & \text { um-hm-uh-hm 1 } & \\ \text { hhn 1 } & \text { oh-okay 2 } & \text { umm 5 } & \\ \text { hmm 2 } & \text { oh-yeah 1 } & \text { ummum 1 } & \end{array}$

Tab. 4: Vorkommen und Häufigkeit nicht-lexikalischer Laute in einem mittelgroßen Korpus amerikanischer Alltagsgespräche (Ward 2006, S. 130)

Im Folgenden soll diese alternative Perspektive auf Interjektionen mit Beispielen aus unserer Forschung auf den Punkt gebracht werden.

\section{Statt Interjektionen: „Lautobjekte“ in ihren sequenziellen und interaktionalen Umgebungen}

Unsere Arbeit zielte darauf ab, Antworten auf die Fragen zu finden: Wann und wie werden Vokalisierungen ${ }^{5}$ von ,echten ${ }^{6}$ Sprechern unter natürlichen Bedingungen systematisch verwendet? Wie werden sie rezipiert? Damit sollte der mangelnde Kenntnisstand über die Formen und den Gebrauch

",“ = starke Grenze zwischen Silben, Wiederholung eines Buchstaben = Längung bzw. schwache Grenze zwischen Silben, „gh“ = velarer Frikativ, „chh“ = palataler Frikativ, „оO“ = /u/ (Ward 2006, S. 138).

4 Wohl ist auch diese Tabelle im Bezug auf Prosodie, erweiterte Phonetik und stimmliche Realisierung defizitär.

5 In der Gesprächsanalyse werden Interjektionen als Vokalisierungen (Goffman 1978) bezeichnet, was für die folgende Darstellung übernommen werden soll. ,Vokalisierung' gilt allerdings hier als Oberbegriff für sämtliche mit den menschlichen Sprechorganen hergestellten Laute und Geräusche. Damit werden sowohl lexikalisierte Interjektionen (wie oben eingeführt) als auch Produktionen jenseits des phonologischen Systems einer Sprache erfasst. In dieser Verwendungsweise schließt Vokalisierung sowohl vokalische als auch nichtvokalische Laute ein. 
von Interjektionen und sonstigen systematisch verwendeten Vokalisierungen gefüllt werden. Um die Kategorie der rekurrent vorkommenden und kommunikativ wirksamen Vokalisierungen zu erfassen, wird der Begriff „Lautobjekt“ eingeführt (Reber 2008).

In unseren Daten treten Vokalisierungen systematisch am häufigsten als Reaktion auf eine Mitteilung auf:

A: Mitteilung

B: Vokalisierung

Mit solchen Mitteilungen können Sprecher verschiedene Handlungen auf der funktionalen Ebene durchführen, z.B. über eine Neuigkeit informieren, ein Verständnisproblem beheben (Reparatur) oder einen Vorschlag ablehnen. Entsprechend können Rezipienten mit Vokalisierungen derartige Handlungen ratifizierend aufnehmen und gleichzeitig ihre affektive Haltung dazu darstellen.

Die Arbeiten von Heritage (1984a) über Mitteilungen, die mit ob aufgenommen werden, sind für unsere Untersuchung richtungsweisend. Im Gegensatz zu Heritage beschränkt sich unsere Arbeit jedoch auf Vokalisierungen, die eine eigene Turnkonstruktionseinheit bilden, und schließt jegliche Art von solchen Vokalisierungen in dieser sequenziellen Position ein. Ähnlich wie bei Heritage und bei Ward (2006) wird ein gebrauchsbasierter Ansatz, von unten'verfolgt: Unsere DATENGRUNDLAGE stützt sich auf ein Korpus von natürlich vorkommenden Gesprächen, d.h. Gesprächen, die auch unabhängig von der Aufnahme für Forschungszwecke stattgefunden hätten. Das Korpus umfasst englische Alltagsgespräche zwischen Freunden und Familienangehörigen und Radiosendungen mit Hörerbeteiligung.

Unser METHODISCHES VORGEHEN orientiert sich am methodologischen Ansatz der Interaktionalen Linguistik (Couper-Kuhlen/Selting (Hg.) 1996; Selting/Couper-Kuhlen 2000, (Hg.) 2001). Für unseren Forschungsgegenstand heisst das, dass Vokalisierungen sowohl formal als auch funktional im Bezug auf ihren Beitrag zur Interaktion betrachtet werden. Daraus folgt unsere spezifische Fragestellung: Welche lautlichen FORMEN und welche sequenziell-organisatorischen und interaktionalen FUNKTIONEN haben VOKALISIERUNGEN, mit denen REZIPIENTEN auf eine MITTEILUNG reagieren? Im folgenden werden drei Thesen vorgestellt, die sich unmittelbar aus unseren Forschungsergebnissen ableiten lassen.

\section{These 1: Manche Lautobjekte bilden Lexeme, ${ }^{6}$ deren Form eine kon- ventionalisierte prosodische Lautgestalt einschließt.}

Diese These soll anhand der verschiedenen Formen und Verwendungen der Vokalisierungen, die im Englischen allgemein mit dem Graphem ob oder 0 verschriftlicht sind, erläutert werden. Unser erstes Fallbeispiel be-

\footnotetext{
Für den Begriff des Lexems siehe u.a. Bauer (1983).
} 
schäftigt sich mit einem so genannten ,extra hohen und spitzen' oh, mit dem Sprecher auf eine Reparatur mit unerwartetem Inhalt, also die Klärung eines Verständnisproblems auf unerwartete Weise, affektiv reagieren können.

In diesem Telefongespräch erkundigt sich Leslie, eine Lehrerin, bei ihrer Kollegin Robin, wie es Mary, einer dritten, unter Allergien leidenden Kollegin, geht. Robin berichtet, dass Mary neue Kleider trägt, die ihr gut stehen.

\section{(1) „Allergies“ [Holt:M88:1:5]}

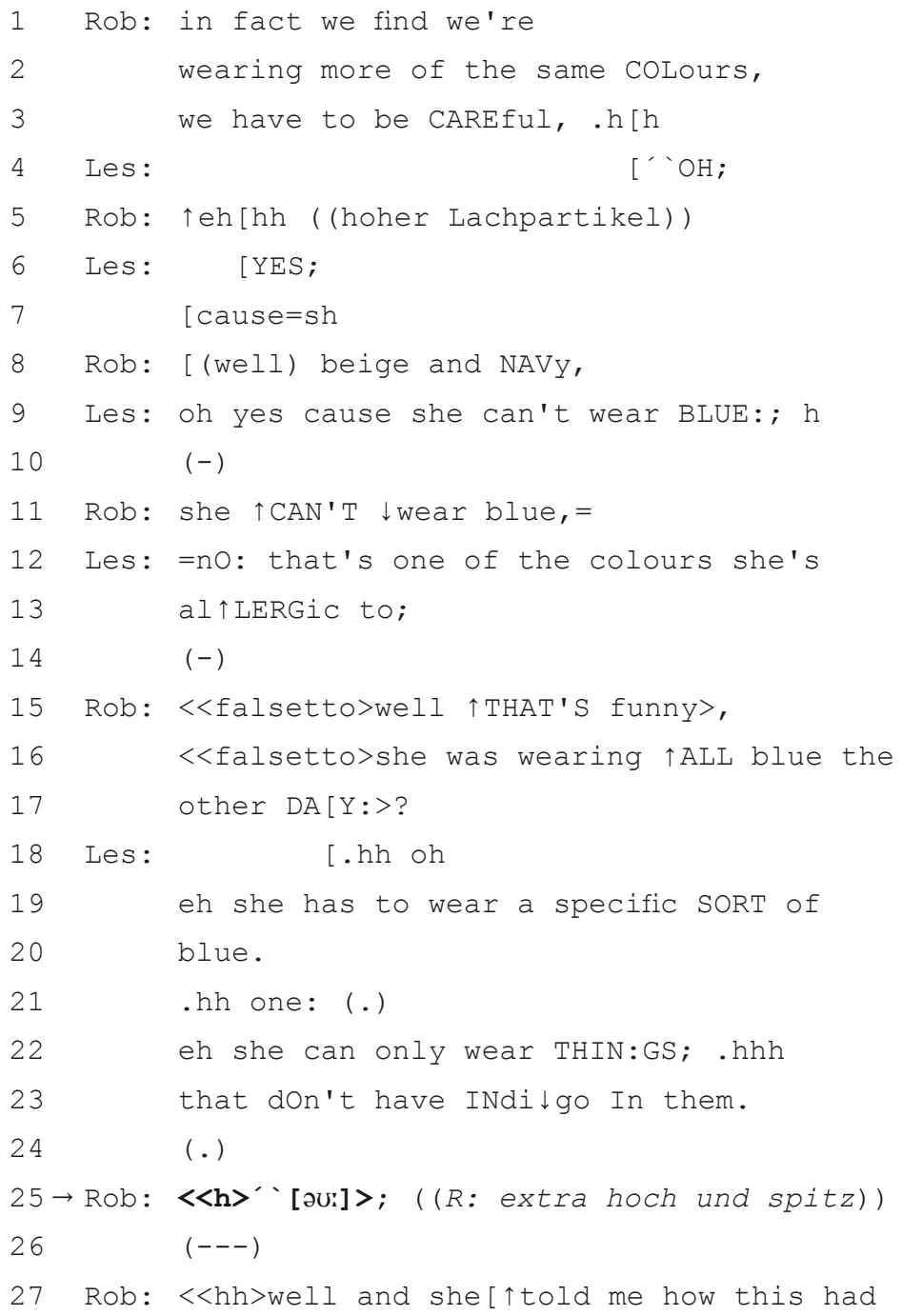




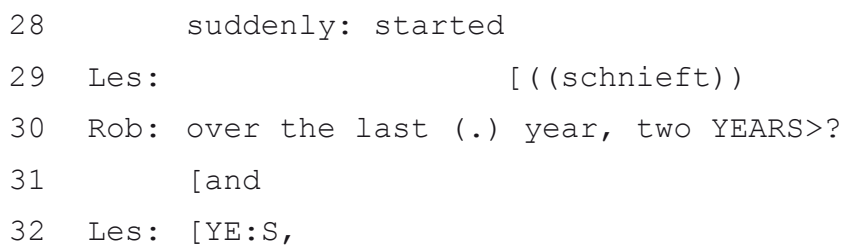

Das ob in Z. 25 bildet aufgrund seiner prosodisch-phonetischen Form ein Beispiel für ein ,extra hohes und spitzes $o b^{6}$ (Reber 2008): Die SEGMENTALE SUBSTANZ besteht aus dem Diphtong [əU]. Weiterhin werden derartige obs mit einer PROSODISCH-PHONETISCHEN FORM realisiert, die sich durch eine Reihe obligatorischer Eigenschaften auszeichnet: Das ob ist gelängt, wird auf einer global sehr hohen, steigend-fallenden Tonhöhenbewegung produziert und folgt speziell in Reparatursequenzen auf den nächsten Schlag. Außerdem weist dieses ob eine große artikulatorische Stärke auf (siehe Tabelle 5).

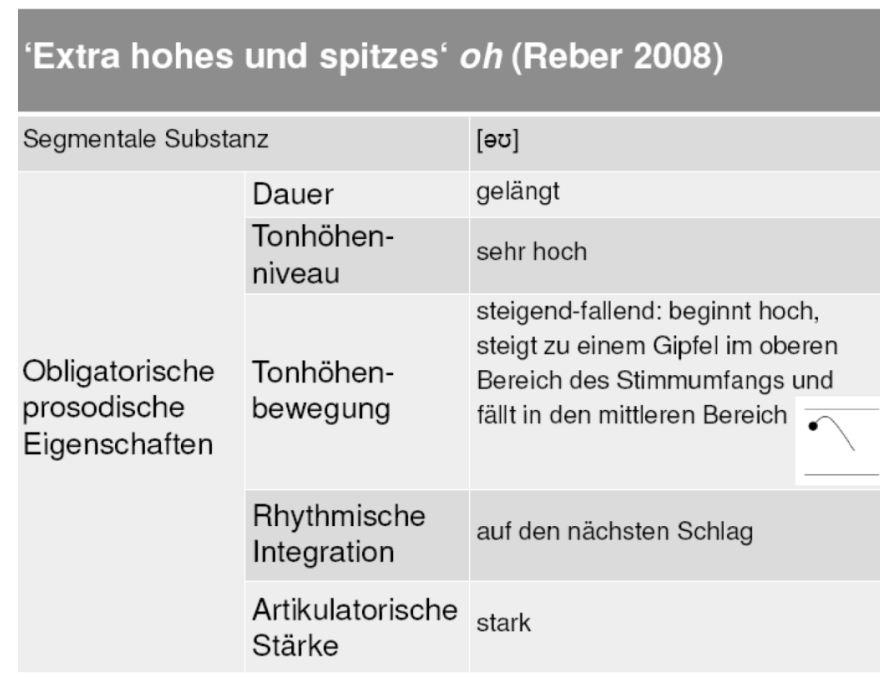

Tab. 5: ,Extra hohes und spitzes` ob (Reber 2008)

Die fakultativen Merkmale umfassen eine höhere Lautstärke als im vorherigen Beitrag derselben Sprecherin, einen initialen Glottalverschluss und behauchte Stimmqualität.

Die SEQUENZANALYSE kommt zu folgendem Ergebnis: Robin agiert in diesem Ausschnitt als Nachrichtenproduzentin über die gemeinsame Kollegin Mary. Robin teilt Leslie mit, dass sie und Mary vorsichtig sein müssen: Sie trügen in letzter Zeit beide Kleidung in denselben Farben, nämlich in 
beige und marineblau (Z. 1-3, 8). In Z. 9 produziert Leslie dagegen den Einwand, dass Mary gar kein Blau tragen kann. Im Gegenzug zeigt Robin Ungläubigkeit über Leslies Mitteilung an und initiiert eine Reparatursequenz (Z. 11). Leslie vollzieht die Reparatur in Z. 18-23, indem sie erklärt, dass Mary nur ein Blau tragen kann, das kein Indigo, einen Farbstoff, enthält. Robin produziert darauf ein extrahohes und spitzes oh (Z. 25). Das Lautobjekt erfüllt an dieser Stelle zwei Funktionen: 1) Eine SEQUENZ-ORGANISATORISCHE FUNKTION, indem mit ihm die Reparatur aufgenommen und ratifiziert wird. 2) Eine INTERAKTIONALE FUNKTION, indem die ob-Produzentin mit dem Lautobjekt ,Überraschung' darstellt. Die Evidenz für diese Feststellung erfolgt sequenzanalytisch. Da die Reparatur aufgrund von abweichenden Erwartungen bzgl. des Reparandums (siehe Z. 11 bzw. 15-17) initiiert wurde, kann geschlossen werden, dass die Reparatur auf der inhaltlichen Ebene unerwartet ist. Eine affektive Reaktion auf eine unerwartete Mitteilung wurde in der gesprächsanalytischen Literatur vielfach als Darstellung von ,Überraschung ${ }^{6}$ beschrieben (,the public display of finding something counter to expectation", Wilkinson/Kitzinger 2006, S. 152; siehe auch Selting 1996; Schegloff 1998). Das, extra hohe und spitze $o b^{6}$ dient also zur überraschten Reparaturaufnahme.

Im nächsten Beispiel wird $o b$ in Form eines zweiten Lautobjekts produziert, nämlich des so genannten ,gedämpften ${ }^{\prime} o h$. Das ,gedämpfte $o b$ wird unter anderen von Sprechern verwendet, um Mitteilungen auf affektive Weise aufzunehmen, mit denen der Gesprächspartner einen Vorschlag oder eine Bitte ablehnt. In unseren Daten kann diese Art von ob als Darstellung von ,Enttäuschung' interpretiert werden.

Bei Beispiel 2 handelt es sich um einen Ausschnitt aus einem Telefongespräch zwischen Leslie und ihren Mann Skip. Leslie macht ihrem Mann den Vorschlag, ihn auf einer Geschäftsreise zu begleiten. Er lehnt jedoch ab, was Leslie mit einem, gedämpften' ob aufnimmt.

(2) „Other chap“ (Holt Sept-Oct 1988 II)

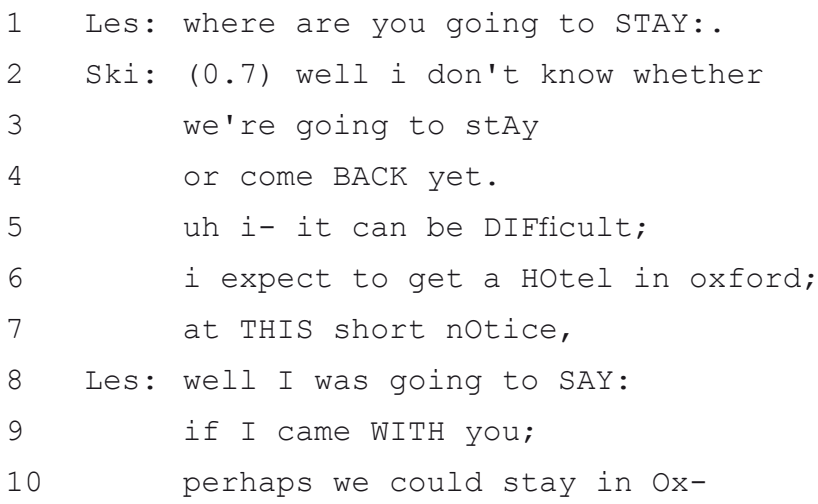




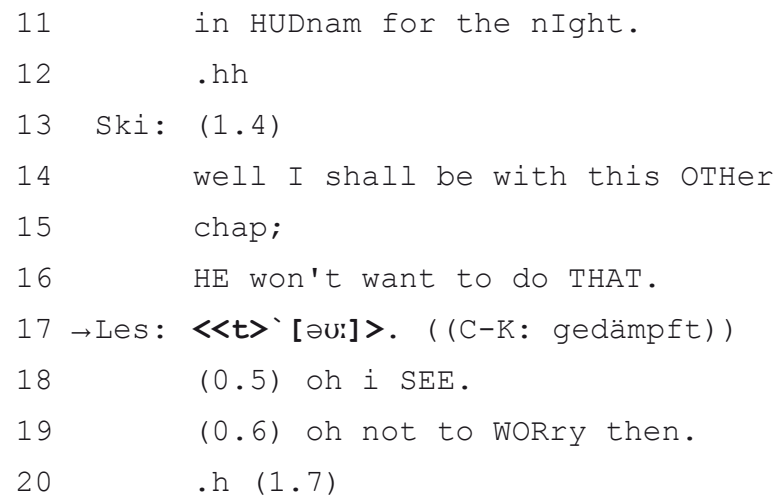

Die lautliche Form des so genannten, gedämpften $o b^{\varsigma}$ (Couper-Kuhlen im Erscheinen a) in Z. 17 kann wie folgt beschrieben werden: Während es in der SEGMENTALEN SUBSTANZ dem extrahohen und spitzen $o b$ ähnelt, unterscheidet es sich in seiner PROSODISCH-PHONETISCHEN FORM: Das ,gedämpfte $o b$ ist gelängt, hat ein tiefes Tonhöhenniveau und wird mit einer geringen und leicht fallenden Tonhöhenbewegung produziert. Es wird leiser als üblich, weder zu früh noch zu spät, mit schwacher artikulatorischer Stärke produziert. Die Phonation ist behaucht und weich im Auslaut (,trailoff', Local/Kelly 1986) (siehe Tabelle 6).

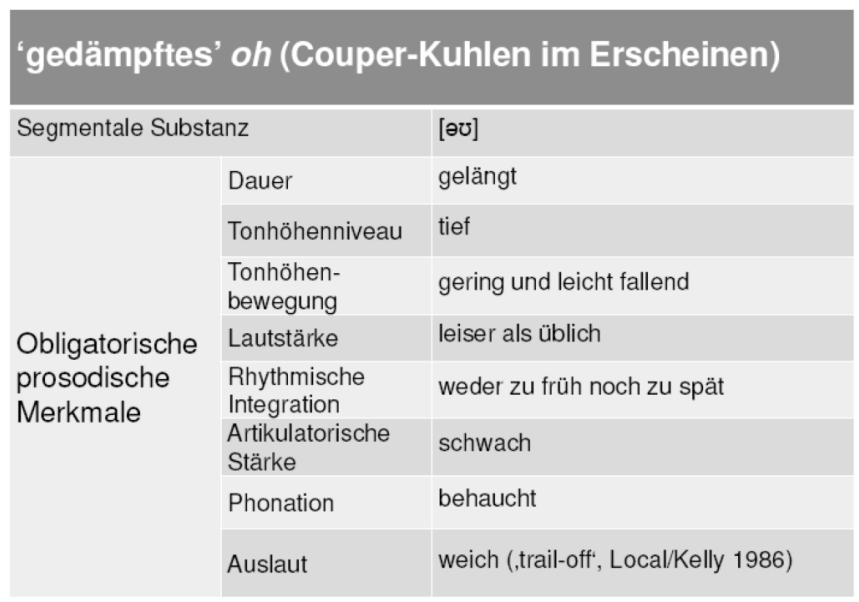

Tab. 6: ,Gedämpftes` oh (Couper-Kuhlen im Erscheinen a)

Die SEQUENZANALYSE lautet wie folgt: Leslie macht in Z. 8-11 den Vorschlag, zusammen mit ihrem Mann in einem Ort namens Hudnam zu übernachten. Nach einer langen Pause lehnt Skip dies ab, indem er darauf verweist, dass sein Kollege dort nicht übernachten wolle (Z. 14-16). Leslie nimmt die Ablehnung mit einem, gedämpften' ob auf (Z. 17). Das Laut- 
objekt erfüllt sowohl eine SEQUENZ-ORGANISATORISCHE FUNKTION als auch eine INTERAKTIONALE FUNKTION: Der Gesprächskontext (konkret: die Sequenz Angebot - Ablehnung - ob) und die prosodischphonetische Realisierung des, gedämpften ${ }^{6}$ ob lassen eine Interpretation dieses ob als Ratifizierungssignal der Ablehnung, das gleichzeitig ,Enttäuschung' anzeigt, zu.

Es wurde verdeutlicht, dass das Graphem ob in Form von verschiedenen Lautobjekten produziert werden kann. Sprecher verwenden die unterschiedlichen Lautobjekte in verschiedenen sequenziellem Kontexten, um unterschiedliche Handlungen mit unterschiedlicher affektiver Qualität zu vollziehen.

Die eben gezeigten Ausschnitte stammen aus Telefongesprächen, in denen die Teilnehmer/innen gefordert waren, allein über ihre Stimme zu kommunizieren, um Emotionen darzubieten. Untersucht man nun Videoaufnahmen von Interaktionen von Angesicht zu Angesicht, entdeckt man, dass solche Vokalisierungen kein ausschließlich lautliches Phänomen sind, sondern als Teil einer körperlichen Gestalt enaktiert werden. Deshalb lautet unsere zweite These:

\section{These 2: Lautobjekte werden als Teil einer körperlichen Gestalt pro- duziert.}

Diese These soll anhand eines Fallbeispiels erläutert werden, in dem ein schmerzerfüllter Laut in Reaktion auf die Ablehnung einer Bitte körperlich enaktiert wird.

Bei diesem Ausschnitt handelt es sich um eine Aufnahme eines Tischgesprächs in einer Kleinfamilie. Anwesend sind Mom, ihr erwachsener Sohn Wesley, seine Verlobte Prudence sowie Virginia, die jüngste Tochter im Teenageralter. Für unsere Analyse zentral ist die Sequenz, in der Virginia ihre Mutter bittet, ein gewisses Sommerkleid aus deren Boutique zu bekommen (Z. 1, 3-4). Die Mutter lehnt die Bitte ab, was von der Tochter unter anderen mit einem Schmerzenslaut (Z. 13) und einem Schnalzlaut (Z.16) aufgenommen wird.

(3) „Summer dress“ [Virginia 00:01:27-00:01:48min]

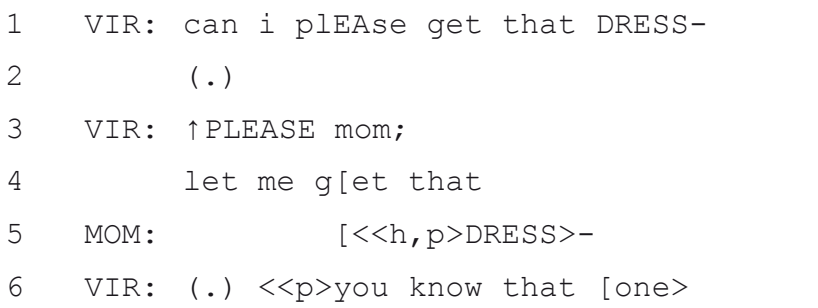




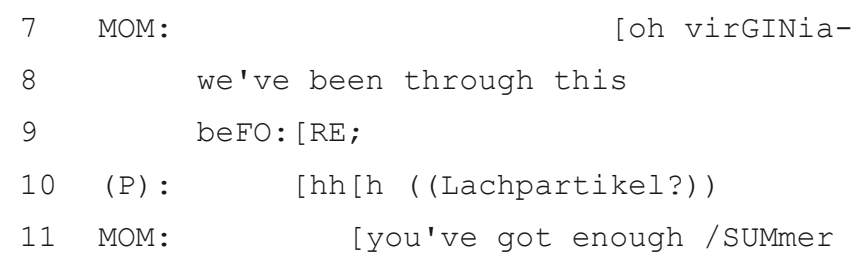

Zunächst zur formalen Analyse des Schmerzenslauts (Z. 13): Die SEGMENTALE SUBSTANZ lässt sich als aspirierter Schwa ([əh]) beschreiben. Die PROSODISCH-PHONETISCHE FORM umfasst folgende Eigenschaften: eine Längung, ein tiefes Tonhöhenniveau, eine flache und leicht fallende Kontur und eine geringe Lautstärke. Der Laut kommt verfrüht, ist von

$\overline{7} \quad$ Das phonetische Symbol stellt einen (post)alveolaren Schnalzlaut dar. 
schwacher Artikulationsstärke, ist behaucht und weich im Auslaut (,trailoff', Local/Kelly 1986). Gleichzeitig gibt die Sprecherin konkordante VISUELL-RÄUMLICHE SIGNALE: Sie wendet ihren Kopf ab und richtet den Blick nach unten.

Der alveolare Schnalzlaut (Z. 16) wird dagegen weder zu früh noch zu spät produziert und folgt auf eine visuell-räumliche Gestalt, in der die Teilnehmerin erneut ihren Kopf abwendet und die Augen niederschlägt (siehe Tabelle 7).

\begin{tabular}{|c|c|c|c|}
\hline \multicolumn{4}{|c|}{ Schmerzerfüllter Laut / Schnalzlaut } \\
\hline \multicolumn{2}{|c|}{ Segmentale Substanz } & [əh] & {$[!]$} \\
\hline \multirow{8}{*}{$\begin{array}{l}\text { Prosodische } \\
\text { Merkmale }\end{array}$} & Dauer & gelängt & - \\
\hline & Tonhöhenniveau & tief & - \\
\hline & $\begin{array}{l}\text { Tonhöhen- } \\
\text { bewegung }\end{array}$ & $\begin{array}{l}\text { gering und leicht } \\
\text { fallend }\end{array}$ & - \\
\hline & Lautstärke & leiser als üblich & - \\
\hline & $\begin{array}{l}\text { Rhythmische } \\
\text { Integration }\end{array}$ & zu früh & $\begin{array}{l}\text { weder zu früh } \\
\text { noch zu spät }\end{array}$ \\
\hline & $\begin{array}{l}\text { Artikulatorische } \\
\text { Stärke }\end{array}$ & schwach & - \\
\hline & Phonation & behaucht & - \\
\hline & Auslaut & $\begin{array}{l}\text { weich (,trail-off', } \\
\text { Local/Kelly 1986) }\end{array}$ & - \\
\hline \multicolumn{2}{|c|}{ Visuelle-räumliche Merkmale } & $\begin{array}{l}\text { Abwenden des } \\
\text { Kopfes, Blick } \\
\text { nach unten }\end{array}$ & $\begin{array}{l}\text { (Abwenden des } \\
\text { Kopfes, Blick } \\
\text { nach unten) }\end{array}$ \\
\hline
\end{tabular}

Tab. 7: Schmerzerfüllter Laut und Schnalzlaut

Unsere SEQUENZANALYSE kommt zu folgendem Ergebnis:

Die Tochter nutzt den Moment, in dem die Mutter ihr den Brotkorb reicht, geschickt aus, um sie um ein Sommerkleid aus deren Laden (Z. 1, 3-4) zu bitten. Da sich beide Teilnehmerinnen zueinander zuwenden, hat sich zu diesem Zeitpunkt der physische Abstand zwischen den beiden Interagierenden verringert. Die Mutter lehnt die Bitte auf emotionale Weise ab (Z. 7-9, 11-12). Virginia reagiert auf die Ablehnung, indem sie sich zunächst auf ihre Ausgangsposition zurückzieht und dann die physische Distanz durch das Abwenden des Kopfes noch weiter vergrößert. Das Abwenden des Kopfes wird durch ein gequältes Ausatmen begleitet. Diese lautlich-visuelle Aufnahme kann als Ratifikation der Ablehnung und gleichzeitig als Darstellung von ,Frustration“ interpretiert werden (vgl. Couper-Kuhlen im Erscheinen b). Sie verläuft simultan mit der Rede der Mutter, die ihren Turn mit einem Angebot fortsetzt (Z. 14-15).

Auch das Angebot wird erneut mit einer lautlich/visuell-räumlichen Aufnahme aufgenommen: Nach kurzen Blickkontakt zur Mutter wendet Virginia wieder den Kopf ab und vergrößert damit die physische Distanz 
zur Mutter. Darauf folgt der Schnalzlaut. Wie im ersten Fall kann das verkörperte Lautobjekt als Ratifizierung der verbalen Mitteilung der Mutter interpretiert werden. Gleichzeit signalisiert die Rezipientin eine affektive Haltung zur Mitteilung, auch diesmal ,Frustration“.

Es wurde deutlich, dass Sprecher Ablehnungen von Bitten in Gesprächen von Angesicht zu Angesicht mit stimmlich/visuell-räumlichen Darstellungen auf affektive Weise ratifizieren können. In diesen Fällen wird das Lautobjekt zum Teil einer visuell-räumlichen Gestalt, die als ganzheitliches Signal wahrgenommmen wird.

Unsere nächste These vertieft die Beobachtung noch weitergehend:

\section{These 3: Manche nicht-lexikalisierten Lautobjekte können ähnliche Funktionen wie lexikalisierte Lautobjekte (d.h. die affektgeladene Aufnahme einer Mitteilung) übernehmen. ${ }^{8}$}

Die dritte These soll anhand eines Fallbeispiels über einen Pfeiflaut (Reber 2008) erläutert werden, der als Reaktion auf eine Nachricht, in der eine Zahl genannt wird, produziert wird. Das Beispiel stammt aus einem Telefongespräch zwischen Mark und seiner Kusine Hilary. In diesem Gespräch teilt Hilary Mark mit, dass ihre Tochter und ihr zukünftiger Schwiegersohn Mike gerade dabei sind, ein gemeinsames Haus zu kaufen. Mike will dabei die Übertragung des Eigentums eigenständig erledigen, weil er damit 1800 Pfund spart. Mark nimmt diese gute Nachricht mit einem Pfeiflaut auf (Z.11).

(4) „conveyancing“ [Holt:M88:2:4]

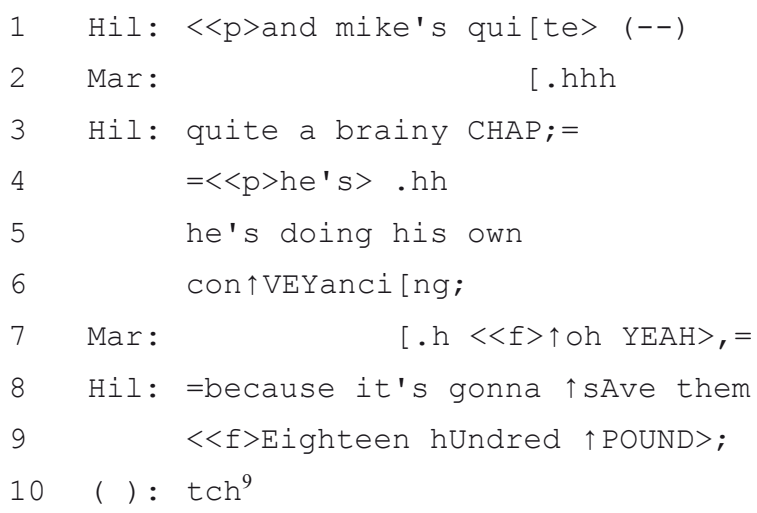

$8 \quad$ Für den Begriff der Lexikalisierung siehe Bauer (1983).

9 Dieser Laut wurde von Gail Jefferson im Originaltranskript dem Rezipienten Mark zugeordnet und als „tch“ notiert. Auf der Grundlage der vorliegenden Audioaufnahme ist der Laut als Schnalzlaut hörbar, jedoch lässt die Qualität der Aufnahme keine nähere Bestimmung des Artikulationsorts noch eine Sprecherzuordnung zu. Da der Schnalzlaut und der Pfeiflaut sehr eng aufeinanderfolgend produziert werden, erscheint es zweifelhaft, ob beide Laute von 


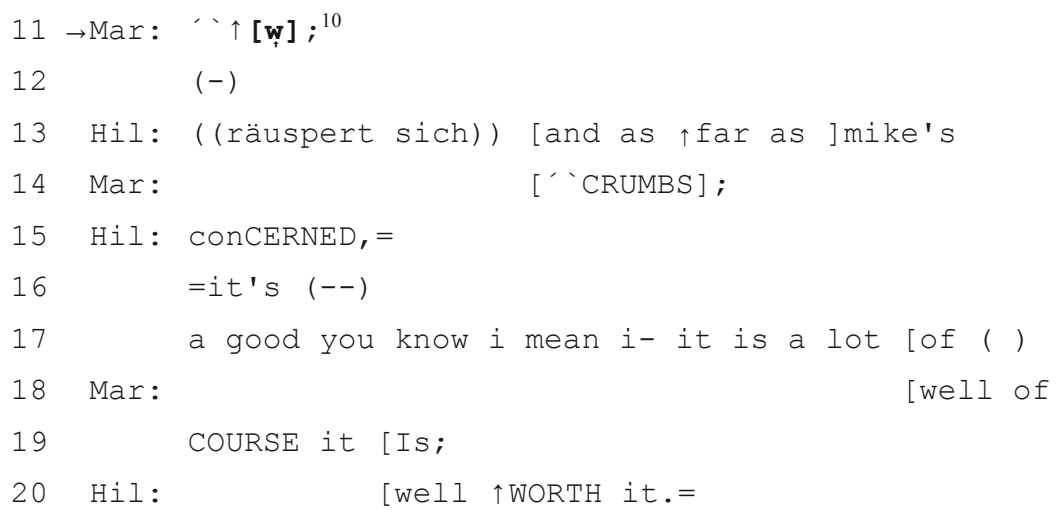

Die SEGMENTALE SUBSTANZ des Pfeiflauts ist in der Literatur als ,recreational labial whistling" (Shosted 2006, S. 2; vgl. auch Shadle 1997, S. 53) beschrieben worden. „Recreational whistling“ unterscheidet sich z.B. von gepfiffenen Frikativen mit Phonemfunktion, wie sie u.a. in einigen Südbantu-Sprachen auftreten. Es zeichnet sich durch ein sehr geringes Reibungsgeräusch aus und kann in einem Frequenzumfang von $500-4000 \mathrm{~Hz}$ auftreten (Shadle 1997, S. 53). Bzgl. der Funktionen konstatiert Shosted ebenso vage wie eindeutig: „Recreational labial whistling is the familiar whistle used to produce a melody or serve any number of paralinguistic functions" (2006, S. 2).

PROSODISCHE MERKMALE des Lautes bilden eine Längung, eine sehr hohe und hoch fallende Tonhöhenbewegung, eine sehr hohe Lautstärke, eine rythmische Integration auf den nächsten Schlag und ein weicher Auslaut (,trail-off“, Local/Kelly 1986). Auch wenn eine Videoaufnahme des Telefonats nicht vorliegt, kann bezüglich VISUELL-RÄUMLICHER MERKMALE davon ausgegangen werden, dass der Produzent beim Pfeifen die Lippen spitzt (siehe Tabelle 8).

Zur SEQUENZANALYSE: Die Nachricht, die mit dem Pfeiflaut (Z. 11) aufgenommen wird, wird als Pointe einer kleinen Erzählung über den Hauskauf dargeboten: Indem die Sprecherin die genaue Summe nennt (Z. 8-9), begründet sie, warum die Hauptfigur, Mike, die Übertragung der Eigentumsrechte selbst übernimmt (Z. 5-6). Außerdem liefert die Sprecherin Evidenz für ihre anfängliche Behauptung in Z. 1 und 3, dass ihr zukünftiger Schwiegersohn ein intelligenter Kerl ist. ${ }^{11}$

ein und demselben Produzenten stammen. Aufgrund dieser Unsicherheiten wird der Schnalzlaut nicht in die Analyse mit einbezogen.

10 Dieses phonetisches Zeichen stellt einen Pfeiflaut (,,recreational labial whistling“) dar.

11 Durch dieses Beispiel wird zudem klar, dass Lautobjekte nicht nur in der dritten Position (wie in den Beispielen 1-3), sondern auch in der zweiten Position einer Sequenz vorkommen können. 


\begin{tabular}{|c|c|c|}
\hline \multicolumn{3}{|c|}{ Pfeiflaut (Reber 2008) } \\
\hline \multicolumn{2}{|c|}{ Segmentale Substanz } & [w] \\
\hline \multirow{6}{*}{$\begin{array}{l}\text { Prosodische } \\
\text { Merkmale }\end{array}$} & Dauer & gelängt \\
\hline & Tonhöhenniveau & sehr hoch \\
\hline & $\begin{array}{l}\text { Tonhöhen- } \\
\text { bewegung }\end{array}$ & hoch fallend \\
\hline & Lautstärke & laut \\
\hline & $\begin{array}{l}\text { Rhythmische } \\
\text { Integration }\end{array}$ & $\begin{array}{l}\text { auf den nächsten } \\
\text { Schlag }\end{array}$ \\
\hline & Auslaut & $\begin{array}{l}\text { weich (,trail-off', } \\
\text { Local/Kelly 1986) }\end{array}$ \\
\hline \multicolumn{2}{|c|}{ Visuelle-räumliche Merkmale } & (gespitzte Lippen) \\
\hline
\end{tabular}

Tab. 8: Pfeiflaut (Reber 2008)

Wie lexikalisierte Vokalisierungen ist der Pfeiflaut multifunktional: Zum einen kann eine SEQUENZ-ORGANISATORISCHE FUNKTION festgestellt werden: Mit dem Pfeiflaut ratifiziert der Rezipient auf der sequenzorganisatorischen Ebene die Neuigkeit als berichtenswert.

Gleichzeitig wird eine INTERAKTIONALE FUNKTION erfüllt: Mit dem Pfeiflaut signalisiert der Sprecher auf affektive Weise seine, Anerkennung ' ,Wertschätzung', ohne die Neuigkeit eindeutig als positiv oder negativ zu bewerten. Auch die sekundäre Interjektion CRUMBS (Z. 14), mit der der Turn nach einer Pause expandiert wird, ist affektgeladen, signalisiert aber keine wertende Sprechereinstellung. ${ }^{12}$ Die Reaktion der Erzählerin liefert Evidenz für diese Interpretation: Sie produziert die fehlende positive Bewertung selbst (Z. 16-17, 20).

Zusammenfassend zeigt dieses Beispiel, dass Sprecher mit einem Pfeiflaut ebenso wie mit ob emotional auf eine Mitteilung reagieren können. Der Pfeiflaut kann eine ähnliche soziale Handlung in sprachlichen Interaktionen erfüllen wie eine lexikalisierte Vokalisierung.

Die vorgestellten Ergebnisse liefern Evidenz, dass Lautobjekte zur Steuerung sozialer Beziehungen verwendet werden, sie sowohl responsiv wirken als auch den nachfolgenden Interaktionsverlauf beeinflussen und somit interaktiv wirksam sind, ihre Form durch ein Bündel von prosodisch-phonetischen (und visuell-räumlichen) Merkmalen bestimmt wird und dass mit lexikalisierten und nichtlexikalisierten Formen vergleichbare Handlungen ausgeführt werden können.

12 Crumbs ist in dieser (britischen) Verwendung ein schwacher Ausdruck des Erstaunens, der dem amerikanischen gosh ungefähr entspricht. 
Darüber hinaus decken sich unsere Ergebnisse mit allgemeinen Ergebnissen, die in der konversationsanalytischen Literatur zu einer Reihe von Sprachen gemacht worden sind. Neben weiteren Arbeiten zum Englischen (Gardner 2001; Goodwin 1986; Local/Walker 2008; Wiggins 2002) wären Untersuchungen z.B. zum Deutschen (z.B. Golato/Betz 2008; Golato/Fagyal 2008), Finnischen (Sorjonen 2001), Französischen (Drescher 1997, 2003), Italienischen (Müller 1996) und Japanischen (Endo 2007) zu nennen.

\section{3. Über die sprachtheoretische Relevanz von Lautobjekten}

Die Befunde, über die wir hier berichtet haben, lassen sich durch Untersuchungen zu weiteren systematisch verwendeten Vokalisierungen im Gespräch ergänzen. Als Analysekriterien schlagen wir dazu ein Schema wie in Tabelle 9 vor:

\begin{tabular}{|c|c|}
\hline Lautobjekt & \\
\hline Segmentale $\varsigma$ & bstanz \\
\hline & Dauer \\
\hline & Tonhöhenniveau \\
\hline & Tonhöhenbewegung \\
\hline Prosodische & Lautstärke \\
\hline Merkmale & Rhythmische Integration \\
\hline & Artikulatorische Stärke \\
\hline & Phonation \\
\hline & Auslaut \\
\hline Visuelle-räum & he Merkmale \\
\hline Sequenzielle & atzierung \\
\hline Sequenz-orga & isationelle Funktion \\
\hline Interaktionale & unktion \\
\hline Turnexpansio & bzw. Sprecherwechsel \\
\hline
\end{tabular}

Tab. 9: Schema für die Analyse von Lautgestalten

Lautobjekte mögen klein und für einige epiphänomenal sein, aber wie anhand eines solchen Schemas sichtbar wird, hat ihre Verwendung im Gespräch eine Systematik. Und diese Systematik ist nicht unwichtig für die Sprachtheorie. In der Tat haben die Befunde zu Lautobjekten Konsequenzen für die Art und Weise, in der wir uns Sprache vorstellen, d.h. für Modelle des so genannten Sprachsystems und für seine Grundlage. Nicht zuletzt wird somit das über Jahrhunderte mühsam errichetete Gebäude der Sprachwissenschaft durch die Befunde zu Lautobjekten tangiert. Im Folgenden sollen vier Fragen bzw. Fragestellungen angesprochen werden, deren Behandlung dazu anregen kann, unsere Konzeptualisierung von Sprache und vom ,Sprachsystem`zu überdenken. 


\subsection{Welche Einheiten gehören zur Sprache?}

Bislang ist es so, dass nur voll LEXIKALISIERTE Einheiten als minimale, bedeutungstragende Teile der Sprache erachtet werden. Als Voraussetzung für die Lexikalisierung werden eine stabile Bedeutungskomponente sowie eine phonologische und eine graphische Form erachtet. Diese Bedingungen treffen (laut dem Duden Universalwörterbuch von 2007) auf die meistens der von Nübling (2004) aufgeführten prototypischen Interjektionen zu: acb!, ab!, aba!, autsch!, bäh!, brr!, bm!, bibi!, boppla!, bu!, buil, burra!, igitt!, jubu!, na!, nanu!, ob!, obo!, oje!, pab!, pfui!, pub!, tja!, uff!, ub!, ui!. Letztere wären infolgedessen regelrechte Lexeme und würden als solche zur Sprache gehören. Jedoch treffen die Lexikalisierungsbedingungen auf ib!, naja!, oi! und phb! nicht zu: diese vier prototypischen Interjektionen sind im Duden-Universalwörterbuch (2007) nicht aufgeführt. Hier wurde offenbar entschieden, dass die Bedeutung bzw. Graphie dieser Exemplare zu instabil sei. Sie wären infolgedessen keine Lexeme. Was sind sie aber dann?

Es lassen sich in der Tat weitaus mehr Vokalisierungen identifizieren als die im Wörterbuch angegebene Interjektionen - Vokalisierungen, die im Gespräch eine systematische Verwendung genießen, darunter für das Englische z.B. den Schnalzlaut (siehe auch Reber 2008; Wright 2005) und den Pfeiflaut. Letztere sind zwar nicht lexikalisiert, sie sind aber im hohen Maße konventionalisiert bzw. institutionalisiert (Bauer 1983). D.h., sie werden wiedererkannt, ihre Produktionsweise ist z.T. voraussagbar und ihr Kontextualisierungspotenzial ist situativ und kontextbezogen eingrenzbar. Darüber hinaus werden sie für ähnliche Funktionen wie die lexikalisierten Vokalisierungen verwendet. Sollten sie nicht deswegen auch zur Sprache gehören? Aus unserer Sicht sollten diese Laute nicht in die paralinguistische Ecke verbannt werden, sondern als zur Sprache gehörig betrachtet werden. Deshalb ziehen wir bewusst den Terminus „Lautobjekt" vor (Reber 2008). Solche Vokalisierungen stellen GESTALTEN dar, weil sie eine holistische Einheit von prosodisch-phonetischen Eigenschaften auf der einen Seite sowie von visuell-räumlichen Eigenschaften auf der anderen bilden. Und als solche holistische Einheiten erfahren sie eine systematische Verwendung im Gespräch.

\subsection{Was für ein System ist Sprache?}

Mit der Erweiterung der Kategorie Lexem, um Lautobjekte zu erfassen, stellt sich fast zwangsläufig die Frage nach dem Sprachsystem. In der Tat stellen die Befunde zu Lautobjekten im Gespräch die herkömmliche Auffassung der Sprache als geschlossenes System in Frage. Denn Realiät ist, dass manche Lautobjekte lexikalisiert, aber andere nur konventionalisiert sind. Und wiederum andere sind erst auf dem Weg zur Konventionalisierung 
(z.B. im Englischen das Lautobjekt /u:/; Reber 2008). Nicht ohne Grund spricht daher Pompino-Marschall bei interjektionsartigen Äußerungen von einem Kontinuum zwischen „Tierlaut“ und „sprachlicher Artikulation“ (2004). Wir sind daher der Meinung, dass man bei der Sprache allenfalls von einem offenen System sprechen kann, offen im Sinne von Hopper (1987, 1988, 1998), dass es ständig in der Emergenz begriffen ist, und zwar in einer Emergenz, die sich aus rekurrenten Verwendungen im Diskurs ergibt:

The linguist's task is $[\ldots]$ to study the whole range of repetition (,formal recurrences') in discourse, and in doing so to seek out those regularities which promise interest as incipient sub-systems. Structure [...] in this view is not an overarching set of abstract principles, but more a question of a spreading of systematicity from individual words, phrases, and small sets (Hopper 1987, S. 142).

Sowohl lexikalisierte als auch konventionalisierte und sich konventionalisierende Vokalisierungen bilden indes eins dieser ,incipient subsystems“ und verdienen es, als emergente Struktur im Sprachsystem betrachtet zu werden.

\subsection{Welche Rolle spielen prosodische sowie visuell-räumliche Modalitäten bei sprachlichen Einheiten?}

Offen ist das Sprachsystem auch in einer anderen Hinsicht. Die Betrachtung der Lautobjekte als holistische Einheiten, bestehend aus Vokalem und Visuell-räumlichem, zeigt auf, dass sprachliche Zeichen mehr als nur Segmente mit entsprechenden Graphemen sind. Im Falle von Interjektionen und Lautobjekten sind sie zunächst auch PROSODISCHE Gestalten (im Unterschied zu anderen Lexemen im Deutschen und Englischen) - in dem Sinne, dass ihre Produktionsweise (ob laut oder leise, hoch oder tief, steigend oder fallend, gespannt oder ungespannt, verspätet oder unverspätet usw.) starken Beschränkungen unterliegt: ihr prosodisches Format ist innerhalb eines gewissen Rahmens vorhersagbar. Mit anderen Worten: Die Art und Weise, wie die Vokalisierung produziert wird, ist mit einer bestimmten sequenzgebundenen Funktion verknüpft.

Diese lautliche Seite ist aber auch mit einer VISUELLEN verflochten: Z.B. ist die Mundstellung für ob anders als die für $a$ h, und erst recht anders als die für einen Pfeiflaut. Beide, Laut und Verkörperung des Lauts, sind intrinsische Bestandteile der Einheit. ${ }^{13}$ Somit dienen die visuellen (und vielleicht auch räumlichen) Aspekte von sprachlichen Einheiten nicht nur zur Unterstützung und zur Erweiterung von lautlichen Aspekten (Stivers/Sidnell 2005, S. 6); sie sind mit der verbalen Substanz verschmolzen und bilden eine einheitliche Gestalt. Eine Trennung wird auf der Wahrnehmungsebene nicht gemacht; sie kann allenfalls analytisch vorgenommen werden.

13 Die auf physiologische Konstitutionsbedingungen zurückzuführenden visuellen Aspekte eines Lautobjekts sind allerdings nicht mit eher kontingenten Begleiterscheinungen wie, gehobene Augenbrauen' oder, weit geöffnete Augen' gleichzusetzen. 


\subsection{Wie sollen wir Sprache untersuchen/modellieren?}

Es folgt daraus, dass sprachliche Untersuchungen zunächst ganzheitlich zu erfolgen haben: Wie Goodwin sagen würde, auf dem Hintergrund eines „reichen multimodalen Kontextes“ (Goodwin 2002). Fokussierungen auf Verbales, Prosodisches, Gestisches bzw. Räumliches sind selbstverständlich aus analytischen Gründen zulässig, müssen aber immer wieder ins Verhältnis zum Gesamten gesetzt werden. Für Lautobjekte speziell, aber auch für andere sprachlichen Konstrukte - allesamt im hohen Grade indexikalisch ist es unabdingbar, sie im situierten Kontext ihrer Verwendung zu untersuchen. Für das Gespräch heisst das, dass Lautobjekte und andere sprachliche Konstrukte im sequenziellen und interaktiven Zusammenhang zu verorten und zu analysieren sind. Denn nur so ist es möglich, ihre Indexikalität zu fassen. Verallgemeinerungen, z.B. für das Lexikon oder für das Inventar an Konstruktionen, haben dieser Kontextbezogenheit Rechnung zu tragen.

Interjektionen und erst recht Lautobjekte sind bisher von der Sprachwissenschaft aufgrund ihrer vermeintlich fehlenden Integration ins Sprachsystem eher stiefmütterlich behandelt worden. Mit der vorgelegten Auswahl an Fallstudien sollte verdeutlicht werden, dass dies zu Unrecht geschehen ist: Wenn man Lautobjekte auf der Gesprächsebene betrachtet, sind sie in ihrer ganzen Lautlichkeit und Körperlichkeit sehr wohl integriert und zwar in eine Grammatik des Redens im Gespräch. ${ }^{14}$ An bestimmten Stellen im Gespräch dürfen solche Lautobjekte nicht fehlen. Somit kann für ein- und allemal mit ihrem Status als „Dazwischengeworfenes“ abgerechnet werden. Sie sind nicht dazwischengeworfen, sondern in ihrer sequenziellen Gesprächsumgebung im hohem Masse systematisch und funktional.

\section{Literatur}

Ameka, Felix (1992): The meaning of phatic and conative interjections. In: Journal of Pragmatics 18, 2/3, S. 245-271.

Auer, Peter/Couper-Kuhlen, Elizabeth/Müller, Frank (1999): Language in time: The rhythm and tempo of spoken interaction. New York.

Auer, Peter/di Luzio, Aldo (Hg.) (1992): The contextualization of language. Amsterdam.

Bauer, Laurie (1983): English word-formation. Cambridge.

Betz, Emma/Golato, Andrea (2008): Remembering relevant information and withholding relevant next actions: The German token achja. In: Research on Language and Social Interaction 41, 1, S. 55-98.

Bühler, Karl (1982): Sprachtheorie. Ungekürzter Neudruck der Ausgabe Jena 1934. Stuttgart/New York.

14 D.h., sie sind in Bezug auf Handlungssequenzen funktional zu beschreiben. 
Couper-Kuhlen, Elizabeth (im Erscheinen a): A sequential approach to affect: The case of ,disappointment‘. In: Haakana, Markku/Laakso, Minna/Lindström, Jan (Hg.): Talk in interaction - comparative dimensions. (= Studia Fennica Linguistica 14). Helsinki.

Couper-Kuhlen, Elizabeth (im Erscheinen b): On preference in responses to rejection. In: Kärkkäinen, Elise/Du Bois, John W. (Hg.): Stance and affect in interaction: Sequential and dialogic perspectives. (Text and Talk Sonderheft).

Couper-Kuhlen, Elizabeth/Ford, Cecilia E. (Hg.) (2004): Sound patterns in interaction. Cross-linguistic studies from conversation. Amsterdam.

Couper-Kuhlen, Elizabeth/Selting, Margret (Hg.) (1996): Prosody in conversation: Interactional studies. Cambridge.

Cruse, David A./Hundsnurscher, Franz/Job, Michael/Lutzeier, Peter Rolf (Hg.) (2002): Lexikologie: Ein Internationales Handbuch zur Natur und Struktur von Wörtern und Wortschätzen. (= Handbücher zur Sprach- und Kommunikationswissenschaft 21.1). Berlin/New York.

Drescher, Martina (1997): Emotions in discourse. French interjections and their use in discourse. In: Niemeier, Susanna/Dirven, René (Hg.): The language of emotions. Conceptualization, expression, and theoretical foundation. Amsterdam, S. 233-246.

Drescher, Martina (2003): Sprachliche Affektivität. Darstellung emotionaler Beteiligung am Beispiel von Gesprächen aus dem Französischen. (= Linguistische Arbeiten 468). Tübingen.

Duden (2005): Duden. Bd. 4: Die Grammatik. 7., völlig neu erarb. und erw. Aufl. Hrsg. v.d. Dudenredaktion. Mannheim/Leipzig/Wien/Zürich.

Duden (2007): Universalwörterbuch. 6., überarb. u. erw. Aufl. Mannheim/Leipzig/ Wien/Zürich. [Online-Version Langenscheidt Berlin].

Ehlich, Konrad (1986): Interjektionen. (= Linguistische Arbeiten 111). Tübingen.

Endo, T. (2007): Sharedness in comprehension and alignment: A::: in Japanese conversation. Unveröff. Qualifizierungsarbeit. Asian Languages and Cultures, UCLA.

Fries, Norbert (2002): Die Wortart ,Interjektionen‘. In: Cruse/Hundsnurscher/Job/ Lutzeier (Hg.), S. 654-657.

Gardner, Rod (2002): When listeners talk: Response tokens and listener stance. Amsterdam.

Goffman, Erving (1963): Behavior in public places. New York.

Goffman, Erving (1978): Response cries. In: Language 54, S. 787-815.

Golato, Andrea/Betz, Emma (2008): German ach and achso in repair uptake: resources to sustain or remove epistemic asymmetry. In: Zeitschrift für Sprachwissenschaft 27, 1, S. 7-37.

Golato, Andrea/Fagyal, Zsuzsanna (2008): Comparing single and double sayings of the German response token $j a$ and the role of prosody - a conversation analytic perspective. In: Research on Language and Social Interaction 41, 3, S. 1-30. 
Goodwin, Charles (1986): Between and within: alternative treatments of continuers and assessments. In: Human Studies 9, S. 205-217.

Goodwin, Charles (2002): Time in action. In: Current Anthropology 43, S. 19-35.

Gumperz, John Joseph (1982): Discourse strategies. Cambridge.

Heritage, John (1984a): A change-of-state-token and aspects of its sequential placement. In: Atkinson, John Maxwell/Heritage, John (Hg.): Structures of social action. Studies in conversation Analysis. Cambridge u.a., S. 299-345.

Heritage, John (1984b): Garfinkel and ethnomethodology. Cambridge.

Hopper, Paul (1987): Emergent grammar. General session and parasession on grammar and cognition. In: Berkeley Linguistic Society 13, S. 139-157.

Hopper, Paul (1988): Emergent grammar and the a priori grammar postulate. In: Tannen, D. (Hg.): Linguistics in context: Connecting observation and understanding. Norwood, S. 117-134.

Hopper, Paul (1998): Emergent grammar. In: Tomasello, Michael (Hg.): The new psychology of language: Cognitive and functional approaches to language structure. Mahwah, S. 155-175.

Kehrein, Roland/Rabanus, Stefan (2001): Ein Modell zur funktionalen Beschreibung von Diskurspartikeln. In: Germanistische Linguistik 157-158, S. 33-50.

Kessler, Florence (2008): Instant Messaging. Eine neue interpersonale Kommunikationsform. In: Net.worx 52, S. 1-72. [www.mediensprache.net/networx/networx-52.pdf (Stand: Oktober 2009)]

Kowal, Sabine/O'Connell, Daniel C. (Hg.) (2004): Zeitschrift für Semiotik 26, 1 /2 [Sonderband zu Interjektionen].

Linell, Per (2005): The written language bias in linguistics: Its nature, origins and transformations. London/New York.

Local, John (1996): Conversational phonetics: Some aspects of news receipts in everyday talk. In: Couper-Kuhlen/Selting (Hg.), S. 175-230.

Local, John/Kelly, John (1986): Projection and ,silences': Notes on phonetic and conversational structure. In: Human Studies 9, S. 185-204.

Local, John/Walker, Gareth (2008): Stance and affect in conversation: On the interplay of sequential and phonetic resources. In: Text \& Talk 28, 7, S. 723-747.

Müller, Frank E. (1996): Affiliating and disaffiliating with continuers: prosodic aspects of recipiency. In: Couper-Kuhlen/Selting (Hg.), S. 131-176.

Nübling, Damaris (2001): Von ob mein Jesus! zu oje! Der Interjektionalisierungspfad von der sekundären zur primären Interjektion. In: Deutsche Sprache 1, 1, S. $20-45$.

Nübling, Damaris (2004): Die prototypische Interjektion: Ein Definitionsvorschlag. In: Zeitschrift für Semiotik 26, $1 / 2$ [Sonderband zu Interjektionen], S. 11-45.

Pompino-Marschall, Bernd (2004): Zwischen Tierlaut und sprachlicher Artikulation: Zur Phonetik der Interjektionen. In: Zeitschrift für Semiotik 26, 1/2 [Sonderband zu Interjektionen], S. 71-84. 
Reber, Elisabeth (2008): Affectivity in talk-in-interaction: Sound objects in English. Unveröff. Diss. Univ. Potsdam.

Reber, Elisaebth (2009): Zur Affektivität in englischen Alltagsgesprächen. In: Buss, Mareike/Habscheid, Stephan/Jautz, Sabine/Liedtke, Frank/Schneider, Jan (Hg.): Theatralität des sprachlichen Handelns. Eine Metaphorik zwischen Linguistik und Kulturwissenschaften. München, S. 193-215.

Reisigl, Martin (1999): Sekundäre Interjektionen: eine diskursanalytische Annäherung. (= Arbeiten zur Sprachanalyse 33). Frankfurt a.M.

Schegloff, Emanuel A. (1998): Reflections on studying prosody in talk-in-interaction. In: Language and Speech, 41, 3/4, S. 235-263.

Schmidt, Jürgen Erich (2001): Bausteine der Intonation? In: Germanistische Linguistik 157-158, S. 9-32.

Schwitalla, Johannes (2006): Gesprochenes Deutsch. Eine Einführung. 3., neu bearb. Aufl. (= Grundlagen der Germanistik 33). Berlin.

Selting, Margret (1996): Prosody as an activity-type distinctive signalling cue in conversation. The case of so-called , astonished questions' in repair-initiation. In: CouperKuhlen/Selting (Hg.), S. 231-270.

Selting, Margret/Couper-Kuhlen, Elizabeth (2000): Argumente für die Entwicklung einer, interaktionalen Linguistik'. In: Gesprächsforschung - Online-Zeitschrift zur verbalen Interaktion (OZS) 1, S. 76-95. [www.gespraechsforschung-ozs.de/heft2000/ ga-selting.pdf (Stand: Oktober 2009)].

Selting, Margret/Couper-Kuhlen, Elizabeth (Hg.) (2001): Studies in interactional linguistics. Amsterdam.

Shadle, Christine (1997): The aerodynamics of speech. In: Hardcastle, William J./Laver, John (Hg.): The handbook of phonetic sciences. Oxford, UK/Cambridge, MA, S. 33-64.

Shosted, Ryan K. (2006): Just put your lips together and blow? The whistled fricatives of Southern Bantu. [http://linguistics.berkeley.edu/phonlab/annual_report/documents/ 2006/whistleISSP.pdf (Stand: Oktober 2009)].

Sorjonen, Marja-Leena (2001): Responding in conversation. A study of response particles in Finnish. Amsterdam/Philadelphia.

Sornig, Karl (2002): Lexikalische Einheiten mit wortähnlichem Status. In: Cruse/Hundsnurscher/Job/Lutzeier (Hg.), S. 461-465.

Stivers, Tanya/Sidnell, Jack (2005): Introduction: Multimodal Interaction. In: Semiotica 156, S. 1-20.

Ward, Nigel (2006): Non-lexical conversational sounds in American English. In: Pragmatics \& Cognition 14, 1, S. 129-182.

Weinrich, Harald (2005): Textgrammatik der deutschen Sprache. 3., rev. Aufl. Hildesheim.

Wharton; Tim (2003): Interjections, language and the ,showing-saying' continuum. In: Pragmatics \& Cognition 11, S. 39-91. 
Wiggins, Sally (2002): Talking with your mouth full. Gustatory Mmms and the embodiment of pleasure. In: Journal of Language and Social Interaction 35, 3, S. 311-36.

Wilkins, David P. (1992): Interjections as deictics. In: Journal of Pragmatics 18, S. 119 158.

Wilkinson, Sue/Kitzinger, Celia (2006): Surprise as an interactional achievement: Reaction tokens in conversation. In: Social Psychology Quarterly 69, 2, S. 150-182.

Wright, Melissa (2005): Studies of the Phonetics-Interaction Interface: Clicks and interactional structures in English conversation. Unveröff. Diss. Univ. York.

Wundt, Wilhelm (1907): Schallnachahmungen und Lautmetaphern in der Sprache. In: Allgemeine Zeitung (Beilage 40), S. 313-316.

\section{Transkriptionskonventionen in Anlehnung an GAT (Selting et al. 1998)}

\section{Sequenzielle Struktur/Verlaufsstruktur}

[ ]

[ ]

$=$

\section{Pausen}

(.)

Mikropause

$(-),(--),(---)$

(2.85) heiten
Überlappungen und Simultansprechen

schneller, unmittelbarer Anschluß neuer Turns oder Ein-

\section{Sonstige segmentale Konventionen}

and $=$ uh

Verschleifungen innerhalb von Einheiten

$:,::,:::$ :

Dehnung, Längung, je nach Dauer

eh, uh, etc.

Verzögerungssignale, sog. ,gefüllte Pausen“

Abbruch einer Einheit

Lachen

so $(h) \circ$

Lachpartikeln beim Reden

Akzentuierung

akZENT

Primär- bzw. Hauptakzent

akzEnt

Sekundär- bzw. Nebenakzent

ak! ZENT!

\section{Tonhöhenbewegung am Einheitenende}

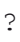

?
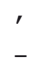

; hoch steigend

mittel steigend

gleichbleibend

mittel fallend

tief fallend 


\section{Auffällige Tonhöhensprünge \\ $\uparrow \quad$ nach oben \\ $\downarrow \quad$ nach unten}

\section{Verändertes Tonhöhenregister}

$\begin{array}{ll}<<\mathrm{t}>> & \text { tiefes Tonhöhenregister } \\ <<\mathrm{h}>> & \text { hohes Tonhöhenregister } \\ <<\mathrm{hh}>> & \text { sehr hohes Tonhöhenregister } \\ <<\text { falsetto }>> & \text { Kopfstimme }\end{array}$

\section{Akzenttonhöhenbewegungen}

$\begin{array}{ll}\text { `so } & \text { fallend } \\ \text { 'so } & \text { steigend } \\ \text { ‘so } & \text { steigend-fallend }\end{array}$

Lautstärke- und Sprechgeschwindigkeitsveränderungen

$$
\begin{array}{ll}
<<\mathrm{f}>> & =\text { forte, laut } \\
<<\mathrm{ff}>> & =\text { fortissimo, sehr laut } \\
<<\mathrm{p}>> & =\text { piano, leise } \\
<<\mathrm{pp}>> & =\text { pianissimo, sehr leise } \\
<<\text { len }>> & =\text { lento, langsam }
\end{array}
$$

\section{Ein- und Ausatmen}

$\begin{array}{ll}. h, . h h, . h h h & \text { Einatmen, je nach Dauer } \\ h, h h, \text { hhh } & \text { Ausatmen, je nach Dauer }\end{array}$

\section{Sonstige Konventionen}

$\begin{array}{ll}\text { ( (hustet) } & \begin{array}{l}\text { para- und außersprachliche Handlungen und Ereignisse, in- } \\ \text { terpretierender Transkribentenkommentar } \\ \text { sprachbegleitende para- und außersprachliche Handlungen } \\ \text { und Ereignisse mit Reichweite }\end{array} \\ \text { ( ) } & \text { unverständliche Passage je nach Länge } \\ \text { ( solche) } & \text { vermuteter Wortlaut } \\ \text { (H) ose } & \text { vermuteter Laut } \\ \rightarrow & \text { Verweis auf im Text behandelte Transkriptzeile } \\ / & \text { Beginn gleichzeitig ablaufender visueller Handlungen }\end{array}$

